Groups Geom. Dyn. 5 (2011), 509-527

DOI $10.4171 / \mathrm{GGD} / 137$
Groups, Geometry, and Dynamics

(C) European Mathematical Society

\title{
Words and mixing times in finite simple groups
}

\author{
Gili Schul and Aner Shalev
}

To Fritz, with fond memory

\begin{abstract}
Let $w \neq 1$ be a non-trivial group word, let $G$ be a finite simple group, and let $w(G)$ be the set of values of $w$ in $G$. We show that if $G$ is large, then the random walk on $G$ with respect to $w(G)$ as a generating set has mixing time 2 .

This strengthens various known results, for example the fact that $w(G)^{2}$ covers almost all of $G$.
\end{abstract}

Mathematics Subject Classification (2010). 20D06, $20 \mathrm{P} 99$.

Keywords. Words, random walks, finite simple groups, mixing time.

\section{Introduction}

Let $w=w\left(x_{1}, \ldots, x_{d}\right)$ be a non-trivial group word, namely a non-identity element of the free group $F_{d}$ on $x_{1}, \ldots, x_{d}$. Then we may write $w=x_{i_{1}}^{n_{1}} x_{i_{2}}^{n_{2}} \ldots x_{i_{k}}^{n_{k}}$ where $i_{j} \in\{1, \ldots, d\}$ and $n_{j}$ are integers. Let $G$ be a group. For $g_{1}, \ldots, g_{d} \in G$ we write $w\left(g_{1}, \ldots, g_{d}\right)=g_{i_{1}}^{n_{1}} g_{i_{2}}^{n_{2}} \ldots g_{i_{k}}^{n_{k}} \in G$. Denote $w(G)=\left\{w\left(g_{1}, \ldots, g_{d}\right) \mid\right.$ $\left.g_{1}, \ldots, g_{d} \in G\right\}$, the set of values of $w$ in $G$. Also, for every subset $A \subseteq G$ we write $A^{k}=\left\{a_{1} \ldots a_{k} \mid a_{i} \in G\right\}$.

An interesting much studied question is how large $w(G)$ is for $G$ a (nonabelian) finite simple group. In [La] it is shown that if $G_{1}, G_{2}, \ldots$ is an infinite sequence of finite simple groups, no two of which are isomorphic, then

$$
\lim _{i \rightarrow \infty} \frac{\log \left|w\left(G_{i}\right)\right|}{\log \left|G_{i}\right|}=1 .
$$

Stronger results were subsequently obtained in [LaSh1] and in [NiPy].

Related Waring type problems were also widely studied, where the goal is to express each group element as a short product of values of $w$; see [LiSh1], [Sh], [Sh1], [LaSh1], [LaSh2], [NiPy]. In [Sh] it is shown that for every group word $w \neq 1$, there exists a positive integer $N=N(w)$ such that for every finite simple group $G$ with $|G| \geq N(w)$ we have $w(G)^{3}=G$. 
In [LaSh1] and [LaSh2] a better result for alternating groups is proved. It is shown that if $w_{1}, w_{2}$ are non-trivial group words, then there exists $N=N\left(w_{1}, w_{2}\right)$ such that for all integers $n \geq N$ we have $w_{1}\left(A_{n}\right) w_{2}\left(A_{n}\right)=A_{n}$.

In this paper we focus on random walks on finite simple groups $G$ with respect to $w(G)$ as a generating set. Our goal is to determine the mixing time of the random walk, namely the time required until we reach an almost uniform distribution on $G$. Our main theorem states that (when $|G|$ is large) this mixing time is the smallest possible, namely 2 .

To make this precise, denote by $U_{G}$ the uniform distribution on $G$, i.e., $U_{G}(g)=$ $\frac{1}{|G|}$ for all $g \in G$. For $W \subseteq G$, denote by $P_{W}$ the uniform distribution on $W$, i.e., $P_{W}(g)=\frac{1}{|W|}$ if $g \in W$ and 0 otherwise.

Denote by $P_{W} * P_{W}$ the convolution of the probability $P_{W}$ with itself. Then $\left(P_{W} * P_{W}\right)(g)$ is the probability that $x y=g$ where $x, y \in W$ are chosen randomly, uniformly and independently.

For two distributions $P, Q$ on $G$ we let

$$
\|P-Q\|_{1}=\sum_{g \in G}|P(g)-Q(g)|
$$

denote the $L_{1}$-distance between $P$ and $Q$.

Theorem 1.1. Fix a word $w \neq 1$, and let $G$ be a finite simple group. Then $\left\|P_{w(G)} * P_{w(G)}-U_{G}\right\|_{1} \rightarrow 0$ as $|G| \rightarrow \infty$.

In fact the same method establishes a similar result for

$$
\left\|P_{w_{1}(G)} * P_{w_{2}(G)}-U_{G}\right\|_{1},
$$

where $w_{1}, w_{2}$ are two non-trivial group words.

This result for alternating groups has recently been obtained in [LaSh2]. It remains to prove it for groups of Lie type, which is what we do here.

From Theorem 1.1 one can deduce that $w(G)^{2}$ covers almost all of $G$ for $G$ a finite simple group and $w$ a non-trivial group word. This has already been proved in Corollary 1.4 of [Sh1].

In fact we prove a more general result of independent interest. Recall that a normal subset of a group $G$ is a subset closed under conjugation (namely a union of conjugacy classes).

If $G$ is a simple group of Lie type then the rank $r$ of $G$ is defined to be the rank of the ambient simple algebraic group, unless we deal with Lie types ${ }^{2} B_{2},{ }^{2} G_{2}$ or ${ }^{2} F_{4}$, in which case $r=1,1,2$, respectively.

Theorem 1.2. Let $G$ be a finite simple group of Lie type of rank $r$ over a field with $q$ elements. Let $W \subseteq G$ be a normal subset. Then for any $\varepsilon>0$ there exists $a$ 
number $R(\varepsilon)$ depending only on $\varepsilon$ such that if $r>R(\varepsilon)$ and $|W| /|G| \geq q^{-r(1-\varepsilon)}$, or if $r \leq R(\varepsilon)$ and $|W| /|G| \geq q^{-(1-\varepsilon)}$, then

$$
\left\|P_{W} * P_{W}-U_{G}\right\|_{1} \rightarrow 0 \quad \text { as }|G| \rightarrow \infty .
$$

Combining this result with known estimates on the size of $w(G)$ we then deduce Theorem 1.1.

In fact we prove a more general result on $k$ normal subsets $W_{1}, \ldots, W_{k}$ and give sufficient conditions for

$$
\left\|P_{W_{1}} * \cdots * P_{W_{k}}-U_{G}\right\|_{1} \rightarrow 0 .
$$

See Theorems 3.3 and 3.5 bellow.

In our proofs we use character theory. To understand the relevance, let $G$ be a finite group, $g \in G$, and let $C_{i}=x_{i}^{G}(i=1, \ldots, k)$ be conjugacy classes. Let $P_{C_{1}, \ldots, C_{k}}(g)$ denote the probability that $y_{1} \ldots y_{k}=g$ where $y_{i} \in C_{i}$ are chosen randomly and uniformly. Notice that $P_{C_{1}, \ldots, C_{k}}=P_{C_{1}} * \cdots * P_{C_{k}}$. Let Irr $G$ denote the set of complex irreducible characters of $G$. It follows from a classical result that

$$
P_{C_{1}, \ldots, C_{k}}(g)=|G|^{-1} \sum_{\chi \in \operatorname{Irr} G} \frac{\chi\left(x_{1}\right) \ldots \chi\left(x_{k}\right) \chi\left(g^{-1}\right)}{\chi(1)^{k-1}} .
$$

For a proof of this result see for instance Theorem 30.4 of [JaLi].

We also use the Witten zeta function $\zeta_{G}$ encoding the character degrees of a finite group $G$. For a real number $s$ define

$$
\zeta_{G}(s)=\sum_{\chi \in \operatorname{Irr} G} \chi(1)^{-s} .
$$

We use the fact established in [LiSh2] that for a finite simple group $G, \zeta_{G}(2) \rightarrow 1$ as $|G| \rightarrow \infty$.

Throughout, $c_{i}$ denote suitable positive absolute constants.

Acknowledgement. We would like to thank the Israel Science Foundation for supporting this research via Grant 754/08.

\section{Preliminaries}

In proving our results we use known estimates for the size of $w(G)$.

The first result in this direction is Proposition 7 of [La]:

Theorem 2.1. For any non-trivial word $w$ and any root system $\Phi$, there exists a constant $c=c(w, \Phi)>0$ such that

$$
|w(G)|>c|G|
$$

for all simple groups $G$ of Lie type associated to the root system $\Phi$. 
Theorem 1.11 of [LaSh1] states:

Theorem 2.2. Let $G$ be a finite simple group of Lie type and of rank $r$. Let $w \neq 1$ be a word. Then if $G$ is not of type $A_{r}$ or ${ }^{2} A_{r}$, we have

$$
|w(G)| \geq c r^{-1}|G|
$$

for some absolute constant $c>0$, provided $|G| \geq N(w)$.

For groups of type $A_{r}$ we use Proposition 1.7 of [NiPy]:

Theorem 2.3. Given $w$ there is a constant $c=c(w)>0$, depending only in $w$, such that if $G=\operatorname{SL}(n, q)$ then

$$
|w(G)|>\frac{c|G|}{n^{3} q^{24+n / 4}} .
$$

For groups of type ${ }^{2} A_{r}$ we use Proposition 1.8 of [NiPy]:

Theorem 2.4. Let $L=\operatorname{SU}(d, q)$. There is a constant $e>0$ such that

$$
|w(L)|>\frac{e|L|}{d^{3} q^{49+d / 4}} .
$$

Using these three results it is easy to deduce the following:

Theorem 2.5. Let $G=G_{r}(q)$ be a finite simple group of Lie type of rank $r$ over the field with $q$ elements. Let $w$ be a group word. There are integers $N=N(w)$ and $R=R(w)$ such that if $|G| \geq N$ and $r \geq R$, then $\frac{|w(G)|}{|G|} \geq q^{-\frac{r}{3}}$.

All these theorems suggest that $w(G)$ is a large normal subset, and we would like to evaluate

$$
\left\|P_{W} * \cdots * P_{W}-U_{G}\right\|_{1}^{2},
$$

for large normal subsets $W$. We can split $W$ into conjugacy classes $C_{i}=x_{i}^{G}$. A classical result states

$$
P_{C_{1}, \ldots, C_{k}}(g)=|G|^{-1} \sum_{\chi \in \operatorname{Irr} G} \frac{\chi\left(x_{1}\right) \ldots \chi\left(x_{k}\right) \chi\left(g^{-1}\right)}{\chi(1)^{k-1}} .
$$

From this we deduce the following lemma, which is probably well known. For completeness we insert a proof.

Lemma 2.6. Let $G$ be a finite group, and let $C_{i}=x_{i}^{G}$ be conjugacy classes. Then

$$
\left\|P_{C_{1}} * \cdots * P_{C_{k}}-U_{G}\right\|_{1}^{2} \leq \sum_{\substack{\chi \in \operatorname{Irr} G \\ \chi \neq 1}} \frac{\left|\chi\left(x_{1}\right)\right|^{2} \cdots\left|\chi\left(x_{k}\right)\right|^{2}}{\chi(1)^{2 k-2}}
$$


Proof.

$$
\begin{aligned}
\left\|P_{C_{1}} * \cdots * P_{C_{k}}-U_{G}\right\|_{1}^{2} & =\left(\left.\sum_{g \in G}\left|P_{C_{1}} * \cdots * P_{C_{k}}(g)-\right| G\right|^{-1} \mid\right)^{2} \\
& =\left(\left.\sum_{g \in G}|| G\right|^{-1} \sum_{\chi \in \operatorname{Irr} G} \frac{\chi\left(x_{1}\right) \ldots \chi\left(x_{k}\right) \chi\left(g^{-1}\right)}{\chi(1)^{k-1}}-|G|^{-1} \mid\right)^{2} \\
& =|G|^{-2}\left(\sum_{g \in G}\left|\sum_{1 \neq \chi \in \operatorname{Irr} G} \frac{\chi\left(x_{1}\right) \ldots \chi\left(x_{k}\right) \chi\left(g^{-1}\right)}{\chi(1)^{k-1}}\right|\right)^{2}
\end{aligned}
$$

By the Cauchy-Schwarz inequality we have,

$$
\begin{aligned}
& |G|^{-2}\left(\sum_{g \in G}\left|\sum_{1 \neq \chi \in \operatorname{Irr} G} \frac{\chi\left(x_{1}\right) \ldots \chi\left(x_{k}\right) \chi\left(g^{-1}\right)}{\chi(1)^{k-1}}\right|\right)^{2} \\
& \leq|G|^{-1} \sum_{g \in G}\left|\sum_{1 \neq \chi \in \operatorname{Irr} G} \frac{\chi\left(x_{1}\right) \ldots \chi\left(x_{k}\right) \chi\left(g^{-1}\right)}{\chi(1)^{k-1}}\right|^{2} \\
& =|G|^{-1} \sum_{g \in G}\left(\sum_{1 \neq \chi \in \operatorname{Irr} G} \frac{\chi\left(x_{1}\right) \ldots \chi\left(x_{k}\right) \chi\left(g^{-1}\right)}{\chi(1)^{k-1}}\right) \overline{\left(\sum_{1 \neq \rho \in \operatorname{Irr} G} \frac{\rho\left(x_{1}\right) \ldots \rho\left(x_{k}\right) \rho\left(g^{-1}\right)}{\rho(1)^{k-1}}\right)} \\
& =|G|^{-1} \sum_{g \in G} \sum_{\chi \neq 1} \sum_{\rho \neq 1} \frac{\chi\left(x_{1}\right) \overline{\rho\left(x_{1}\right)} \ldots \chi\left(x_{k}\right) \overline{\rho\left(x_{k}\right)} \chi\left(g^{-1}\right) \overline{\rho\left(g^{-1}\right)}}{\chi(1)^{k-1} \overline{\rho(1)^{k-1}}} \\
& =|G|^{-1} \sum_{\chi \neq 1} \sum_{\rho \neq 1} \frac{\chi\left(x_{1}\right) \overline{\rho\left(x_{1}\right)} \ldots \chi\left(x_{k}\right) \overline{\rho\left(x_{k}\right)}}{\chi(1)^{k-1} \overline{\rho(1)^{k-1}}}\left(\sum_{g \in G} \chi\left(g^{-1}\right) \overline{\rho\left(g^{-1}\right)}\right) \\
& =\sum_{1 \neq \chi \in \operatorname{Irr} G} \frac{\left|\chi\left(x_{1}\right)\right|^{2} \ldots\left|\chi\left(x_{k}\right)\right|^{2}}{\chi(1)^{2 k-2}} .
\end{aligned}
$$

The last equality is by the orthogonality relations (see e.g. [Ser]).

Now we use known results on the irreducible representations of finite simple groups of Lie type. By [LiSh3] Section 6 (see also Lemma 4.6 of [Sh]) we have:

Lemma 2.7. Let $G=G_{r}(q)$ be a finite simple classical group. Then $\operatorname{Irr} G$ has a subset $W$ of so called Weil characters with the following properties:

(i) $|\mathfrak{W}| \leq q+1$.

(ii) Let $\chi \in \mathcal{W}$ and $x \in G$. If $\left|C_{G}(x)\right| \leq q^{m}$ for some integer $m$, then $|\chi(x)| \leq$ $q^{\sqrt{m+b}}$ where $b$ is some absolute constant.

(iii) If $1 \neq \chi \in \operatorname{Irr} G \backslash \mathcal{W}$ and $r>5$ then $\chi(1) \geq c q^{2 r-3}$ where $c>0$ is some absolute constant. 
We also use the following:

Lemma 2.8. Let $G=G_{r}(q)$ be a finite simple group of Lie type of rank $r$ over the field with q elements, and let $k(G)$ denote the number of conjugacy classes of $G$.

(i) There is a positive constant $c_{1}$ such that $\chi(1) \geq c_{1} q^{r}$ for all $1 \neq \chi \in \operatorname{Irr} G$.

(ii) There is a positive constant $c_{2}$ such that $k(G) \leq c_{2} q^{r}$.

Proof. Part (i) follows from [LanSe] and (ii) from [FuGu].

Theorem 1.1 of [LiSh2] states:

Theorem 2.9. Let $G$ be a finite simple group, and for a real number $s$ let

$$
\zeta_{G}(s)=\sum_{\chi \in \operatorname{Irr} G} \chi(1)^{-s} .
$$

If $s>1$ then $\zeta_{G}(s) \rightarrow 1$ as $|G| \rightarrow \infty$.

We also use known estimates for the number of regular semisimple elements. Recall that an element $x$ of a finite group $G$ of Lie type is called regular if its centralizer in the corresponding algebraic group $\bar{G}$ has minimal dimension, namely $\operatorname{rank}(\bar{G})$.

We say that $x$ is semisimple if its order is not divisible by $p$, where $p$ is the defining characteristic of $G$. The next result is of Guralnick and Lübeck in [GuLu]:

Theorem 2.10. Let $G$ be a finite simple group of Lie type over the field with $q$ elements. Denote by $r(G)$ the proportion of regular semisimple elements in $G$. Then

$$
1-r(G)<\frac{3}{q-1}+\frac{2}{(q-1)^{2}}
$$

From this theorem, using elementary computations, we easily obtain:

Corollary 2.11. Let $G$ be a finite simple group of Lie type over the field with $q$ elements. Denote by $r(G)$ the proportion of regular semisimple elements in $G$. Then

$$
1-r(G)<\frac{5}{q} \text {. }
$$

So we can see that there are many regular semisimple elements, and we use the next lemma when dealing with these elements in groups of bounded rank:

Lemma 2.12. Let $G=G_{r}(q)$ be a finite simple group of Lie type of rank $r$ over the field with $q$ elements, and let $x \in G$ be a regular semisimple element. Then there is a number $c=c(r)$, depending on $r$ but not on $q$, such that $|\chi(x)| \leq c$ for all $\chi \in \operatorname{Irr} G$.

Proof. This follows from the Deligne-Lusztig theory; see [Lus], and formula 4.26.1 in particular. 


\section{Proofs}

Lemma 3.1. Let $G$ be a finite group, $k \geq 2$ an integer, and let $x_{1}, x_{2}, \ldots, x_{k} \in G$ be elements of $G$. Then

$$
\sum_{\chi \in \operatorname{Irr} G}\left|\chi\left(x_{1}\right) \chi\left(x_{2}\right) \ldots \chi\left(x_{k}\right)\right| \leq\left|C_{G}\left(x_{1}\right)\right|^{\frac{1}{2}}\left|C_{G}\left(x_{2}\right)\right|^{\frac{1}{2}} \ldots\left|C_{G}\left(x_{k}\right)\right|^{\frac{1}{2}} .
$$

Proof. By the orthogonality relations (see e.g. [Ser]) we have

$$
\sum_{\chi \in \operatorname{Irr} G}\left|\chi\left(x_{i}\right)\right|^{2}=\left|C_{G}\left(x_{i}\right)\right|
$$

In particular $\left|\chi\left(x_{i}\right)\right| \leq\left|C_{G}\left(x_{i}\right)\right|^{\frac{1}{2}}$ for all $1 \leq i \leq k$ and all $\chi \in \operatorname{Irr} G$.

Clearly

$$
\sum_{\chi \in \operatorname{Irr} G}\left|\chi\left(x_{1}\right) \chi\left(x_{2}\right) \ldots \chi\left(x_{k}\right)\right| \leq\left|C_{G}\left(x_{3}\right)\right|^{\frac{1}{2}} \ldots\left|C_{G}\left(x_{k}\right)\right|^{\frac{1}{2}} \sum_{\chi \in \operatorname{Irr} G}\left|\chi\left(x_{1}\right) \chi\left(x_{2}\right)\right| .
$$

By the Cauchy-Schwarz inequality we have

$$
\begin{aligned}
\sum_{\chi \in \operatorname{Irr} G}\left|\chi\left(x_{1}\right) \chi\left(x_{2}\right)\right| & \leq\left(\sum_{\chi \in \operatorname{Irr} G}\left|\chi\left(x_{1}\right)\right|^{2}\right)^{\frac{1}{2}}\left(\sum_{\chi \in \operatorname{Irr} G}\left|\chi\left(x_{2}\right)\right|^{2}\right)^{\frac{1}{2}} \\
& =\left|C_{G}\left(x_{1}\right)\right|^{\frac{1}{2}}\left|C_{G}\left(x_{2}\right)\right|^{\frac{1}{2}} .
\end{aligned}
$$

The result now follows from the two inequalities above.

Theorem 3.2. Let $G=G_{r}(q)$ be a finite simple group of Lie type of rank $r$ over the field with $q$ elements. Let $k \geq 2$ be an integer. Let $\varepsilon>0$ and let $x_{1}, x_{2}, \ldots, x_{k} \in G$ such that

$$
\prod_{i=1}^{k}\left|C_{G}\left(x_{i}\right)\right| \leq q^{\left(4 k-4-\left(3-\frac{4}{k}\right) \varepsilon\right) r} .
$$

(i) If $r>5$ and $G$ is a classical group, then

$$
\sum_{\substack{\chi \in \operatorname{Irr} G \\ \chi \neq 1}} \frac{\left|\chi\left(x_{1}\right) \ldots \chi\left(x_{k}\right)\right|}{\chi(1)^{k-1}} \leq 2 c_{1}^{1-k} q^{k \sqrt{(4 k-4) r+b}-r(k-1)+1}+c_{2}^{1-k} q^{3 k-3-\left(1.5-\frac{2}{k}\right) r \varepsilon},
$$

where $c_{1}, c_{2}$ and $b$ are absolute constants.

(ii) There exists a real number $r_{1}=r_{1}(k, \varepsilon)$ such that if $r \geq r_{1}$ then

$$
\sum_{\substack{\chi \in \operatorname{Irr} G \\ \chi \neq 1}} \frac{\left|\chi\left(x_{1}\right) \ldots \chi\left(x_{k}\right)\right|}{\chi(1)^{k-1}} \rightarrow 0 \quad \text { as }|G| \rightarrow \infty .
$$


Proof. We first prove (i).

Let $\mathcal{W}$ be the set of Weil characters of $G$ (see 2.7). Set

$$
\sum_{\substack{\chi \in \operatorname{Irr} G \\ \chi \neq 1}} \frac{\left|\chi\left(x_{1}\right) \ldots \chi\left(x_{k}\right)\right|}{\chi(1)^{k-1}}=\sum_{\chi \in \mathcal{W}} \frac{\left|\chi\left(x_{1}\right) \ldots \chi\left(x_{k}\right)\right|}{\chi(1)^{k-1}}+\sum_{\substack{\chi \notin \mathcal{W} \\ \chi \neq 1}} \frac{\left|\chi\left(x_{1}\right) \ldots \chi\left(x_{k}\right)\right|}{\chi(1)^{k-1}} .
$$

We will handle each summand separately.

The first summand:

From our assumptions $\left|C_{G}\left(x_{i}\right)\right| \leq q^{(4 k-4) r}$ and so (ii) of Lemma 2.7 yields $\left|\chi\left(x_{i}\right)\right| \leq q^{\sqrt{(4 k-4) r+b}}$ for $i=1, \ldots, k$ and $\chi \in \mathcal{W}$. We also have $\chi(1) \geq c_{1} q^{r}$ for all non-trivial $\chi \in \operatorname{Irr} G$ (i) of Lemma 2.8. Therefore

$$
\sum_{\chi \in \mathcal{W}} \frac{\left|\chi\left(x_{1}\right) \ldots \chi\left(x_{k}\right)\right|}{\chi(1)^{k-1}} \leq \sum_{\chi \in \mathcal{W}} \frac{q^{k \sqrt{(4 k-4) r+b}}}{\chi(1)^{k-1}} \leq \frac{|\mathcal{W}| q^{k \sqrt{(4 k-4) r+b}}}{c_{1}^{k-1} q^{(k-1) r}} .
$$

From (i) of Lemma 2.7 we have $|\mathcal{W}| \leq q+1$ and so

$$
\begin{aligned}
\sum_{\chi \in W} \frac{\left|\chi\left(x_{1}\right) \ldots \chi\left(x_{k}\right)\right|}{\chi(1)^{k-1}} & \leq \frac{(q+1) q^{k \sqrt{(4 k-4) r+b}}}{c_{1}^{k-1} q^{(k-1) r}} \\
& \leq \frac{2 q \cdot q^{k \sqrt{(4 k-4) r+b}}}{c_{1}^{k-1} q^{(k-1) r}} \\
& =2 c_{1}^{1-k} q^{k \sqrt{(4 k-4) r+b}-(k-1) r+1}
\end{aligned}
$$

The second summand:

If $1 \neq \chi \notin \mathcal{W}$ and $r>5$, (iii) of Lemma 2.7 yields $\chi(1) \geq c_{2} q^{2 r-3}$. Hence

$$
\begin{aligned}
\sum_{\substack{\chi \notin W \\
\chi \neq 1}} \frac{\left|\chi\left(x_{1}\right) \ldots \chi\left(x_{k}\right)\right|}{\chi(1)^{k-1}} & \leq c_{2}^{1-k} q^{(1-k)(2 r-3)} \sum_{\substack{\chi \notin W \\
\chi \neq 1}}\left|\chi\left(x_{1}\right) \ldots \chi\left(x_{k}\right)\right| \\
& \leq c_{2}^{1-k} q^{(1-k)(2 r-3)} \sum_{\chi \in \operatorname{Irr} G}\left|\chi\left(x_{1}\right) \ldots \chi\left(x_{k}\right)\right| .
\end{aligned}
$$

Using Lemma 3.1 we obtain

$$
\sum_{\substack{\chi \notin W \\ \chi \neq 1}} \frac{\left|\chi\left(x_{1}\right) \ldots \chi\left(x_{k}\right)\right|}{\chi(1)^{k-1}} \leq c_{2}^{1-k} q^{(1-k)(2 r-3)}\left|C_{G}\left(x_{1}\right)\right|^{\frac{1}{2}} \ldots\left|C_{G}\left(x_{k}\right)\right|^{\frac{1}{2}} .
$$

Using our assumption on $\left|C_{G}\left(x_{1}\right)\right| \ldots\left|C_{G}\left(x_{k}\right)\right|$ we obtain

$$
\begin{aligned}
\sum_{\substack{\chi \notin W \\
\chi \neq 1}} \frac{\left|\chi\left(x_{1}\right) \ldots \chi\left(x_{k}\right)\right|}{\chi(1)^{k-1}} & \leq c_{2}^{1-k} q^{(1-k)(2 r-3)} q^{\left(2 k-2-\left(1.5-\frac{2}{k}\right) \varepsilon\right) r} \\
& =c_{2}^{1-k} q^{3 k-3-\left(1.5-\frac{2}{k}\right) r \varepsilon} .
\end{aligned}
$$


The sum:

We conclude that for $r>5$ and for $G$ a classical group,

$$
\sum_{\substack{\chi \in \operatorname{Irr} G \\ \chi \neq 1}} \frac{\left|\chi\left(x_{1}\right) \ldots \chi\left(x_{k}\right)\right|}{\chi(1)^{k-1}} \leq 2 c_{1}^{1-k} q^{k \sqrt{(4 k-4) r+b}-(k-1) r+1}+c_{2}^{1-k} q^{3 k-3-\left(1.5-\frac{2}{k}\right) r \varepsilon},
$$

proving (i).

Since the simple groups of Lie type which are not classical are of rank at most 8 , we can use (i) by assuming also $r>8$.

Hence, if $r>8$ and also large enough so that

$$
k \sqrt{(4 k-4) r+b}-(k-1) r+1<0
$$

and

$$
3 k-3-\left(1.5-\frac{2}{k}\right) r \varepsilon<0
$$

then

$$
\sum_{\substack{\chi \in \operatorname{Irr} G \\ \chi \neq 1}} \frac{\left|\chi\left(x_{1}\right) \ldots \chi\left(x_{k}\right)\right|}{\chi(1)^{k-1}} \rightarrow 0 \quad \text { as }|G| \rightarrow \infty
$$

proving (ii).

Theorem 3.3. Let $G$ be a finite simple group of Lie type of rank $r$ over the field with $q$ elements. Let $k \geq 2$ be an integer. Let $0<\varepsilon$. Let $W_{1}, \ldots, W_{k}$ be normal subsets such that

$$
\frac{\left|W_{i}\right|}{|G|} \geq q^{-\left(3-\frac{4}{k}\right) r(1-\varepsilon)} \text {. }
$$

Then there exists a real number $r_{1}=r_{1}(k, \varepsilon)$ such that the following holds.

(i) If $r \geq r_{1}$ and $|G| \geq N=N(k, \varepsilon)$, where $N$ is an integer that depends only on $k$ and $\varepsilon$, then

$$
\begin{aligned}
& \left\|P_{W_{1}} * \cdots * P_{W_{k}}-U_{G}\right\|_{1} \\
& \leq \sqrt{2 c_{1}^{1-k} q^{k \sqrt{(4 k-4) r+b}-r(k-1)+1}+c_{2}^{1-k} q^{3 k-3-\left(1.5-\frac{2}{k}\right) r \varepsilon}} \\
& \quad+\left(2^{k}-1\right) 2 c_{3} q^{-\left(3-\frac{4}{k}\right) \frac{k-1}{k} \varepsilon r}
\end{aligned}
$$

where $c_{1}, c_{2}, c_{3}, b$ are absolute constants.

(ii) If $r \geq r_{1}$ then $\left\|P_{W_{1}} * \cdots * P_{W_{k}}-U_{G}\right\|_{1} \rightarrow 0$ as $|G| \rightarrow \infty$.

Proof. Partition $W_{i}$ into two subsets $W_{i, 1}$ and $W_{i, 2}$ as follows:

$$
\begin{aligned}
& W_{i, 1}=\left\{x \in W_{i}:\left|C_{G}(x)\right| \leq q^{\left(4-\frac{4}{k}-\frac{(3 k-4) \varepsilon}{k^{2}}\right) r}\right\} \\
& W_{i, 2}=\left\{x \in W_{i}:\left|C_{G}(x)\right|>q^{\left(4-\frac{4}{k}-\frac{(3 k-4) \varepsilon}{k^{2}}\right) r}\right\} .
\end{aligned}
$$


Then $W_{i, 1}$ and $W_{i, 2}$ are normal subsets, and

$$
W_{i}=W_{i, 1} \cup W_{i, 2}, \quad W_{i, 1} \cap W_{i, 2}=\phi .
$$

Hence,

It follows that

$$
P_{W_{i}}=\frac{\left|W_{i, 1}\right|}{\left|W_{i}\right|} P_{W_{i, 1}}+\frac{\left|W_{i, 2}\right|}{\left|W_{i}\right|} P_{W_{i, 2}}
$$

$$
\begin{array}{rl}
P_{W_{1}} & * \cdots * P_{W_{k}} \\
& =\left(\frac{\left|W_{1,1}\right|}{\left|W_{1}\right|} P_{W_{1,1}}+\frac{\left|W_{1,2}\right|}{\left|W_{1}\right|} P_{W_{1,2}}\right) * \cdots *\left(\frac{\left|W_{k, 1}\right|}{\left|W_{k}\right|} P_{W_{k, 1}}+\frac{\left|W_{k, 2}\right|}{\left|W_{k}\right|} P_{W_{k, 2}}\right) \\
& =\sum_{\left(j_{1}, \ldots, j_{k}\right) \in\{1,2\}^{k}}\left(\frac{\left|W_{1, j_{1}}\right|}{\left|W_{1}\right|} \ldots \frac{\left|W_{k, j_{k}}\right|}{\left|W_{k}\right|}\right) P_{W_{1, j}} * \cdots * P_{W_{k, j_{k}}} .
\end{array}
$$

Since

$$
U_{G}=\sum_{\left(j_{1}, \ldots, j_{k}\right) \in\{1,2\}^{k}}\left(\frac{\left|W_{1, j_{1}}\right|}{\left|W_{1}\right|} \ldots \frac{\left|W_{k, j_{k}}\right|}{\left|W_{k}\right|}\right) U_{G}
$$

we have

$$
\begin{aligned}
& \left\|P_{W_{1}} * \cdots * P_{W_{k}}-U_{G}\right\|_{1} \\
& \quad \leq \sum_{\left(j_{1}, \ldots, j_{k}\right) \in\{1,2\}^{k}} \frac{\left|W_{1, j_{1}}\right|}{\left|W_{1}\right|} \ldots \frac{\left|W_{k, j_{k}}\right|}{\left|W_{k}\right|}\left\|P_{W_{1, j_{1}}} * \cdots * P_{W_{k, j_{k}}}-U_{G}\right\|_{1} .
\end{aligned}
$$

We will handle the first summand

$$
\frac{\left|W_{1,1}\right|}{\left|W_{1}\right|} \ldots \frac{\left|W_{k, 1}\right|}{\left|W_{k}\right|}\left\|P_{W_{1,1}} * \cdots * P_{W_{k, 1}}-U_{G}\right\|_{1}
$$

differently from the other $2^{k}-1$ summands.

The first summand:

$$
\frac{\left|W_{1,1}\right|}{\left|W_{1}\right|} \ldots \frac{\left|W_{k, 1}\right|}{\left|W_{k}\right|}\left\|P_{W_{1,1}} * \cdots * P_{W_{k, 1}}-U_{G}\right\|_{1} \leq\left\|P_{W_{1,1}} * \cdots * P_{W_{k, 1}}-U_{G}\right\|_{1} \text {. }
$$

$W_{i, 1}$ is a normal subset, and hence is a union of conjugacy classes. Denote the conjugacy classes of $W_{i, 1}$ by $C_{i, 1}, \ldots C_{i, m_{i}}$. So $W_{i, 1}=\bigcup_{j=1}^{m_{i}} C_{i, j}$.

Hence,

$$
\begin{aligned}
P_{W_{1,1}} * \cdots * P_{W_{k, 1}} & =\left(\sum_{j_{1}=1}^{m_{1}} \frac{\left|C_{1, j_{1}}\right|}{\left|W_{1,1}\right|} P_{C_{1, j_{1}}}\right) * \cdots *\left(\sum_{j_{k}=1}^{m_{k}} \frac{\left|C_{k, j_{k}}\right|}{\left|W_{k, 1}\right|} P_{C_{k, j_{k}}}\right) \\
& =\sum_{\substack{1 \leq j_{1} \leq m_{1} \\
1 \leq j_{k} \leq m_{k}}} \frac{\left|C_{1, j_{1}}\right| \ldots\left|C_{k, j_{k}}\right|}{\left|W_{1,1}\right| \ldots\left|W_{k, 1}\right|} P_{C_{1, j_{1}}} * \cdots * P_{C_{k, j_{k}}} .
\end{aligned}
$$


Therefore,

$$
\left\|P_{W_{1,1}} * \cdots * P_{W_{k, 1}}-U_{G}\right\|_{1} \leq \sum_{\substack{1 \leq j_{1} \leq m_{1} \\ 1 \leq j_{k} \leq m_{k}}} \frac{\left|C_{1, j_{1}}\right| \ldots\left|C_{k, j_{k}}\right|}{\left|W_{1,1}\right| \ldots\left|W_{k, 1}\right|}\left\|P_{C_{1, j_{1}}} * \cdots * P_{C_{k, j_{k}}}-U_{G}\right\|_{1} .
$$

According to Lemma 2.6,

$$
\left\|P_{C_{1, j_{1}}} * \cdots * P_{C_{k, j_{k}}}-U_{G}\right\|_{1}^{2} \leq \sum_{\substack{\chi \in \operatorname{Irr} G \\ \chi \neq 1}} \frac{\left|\chi\left(x_{1, j_{1}}\right)\right|^{2} \ldots\left|\chi\left(x_{k, j_{k}}\right)\right|^{2}}{\chi(1)^{2 k-2}}
$$

where $C_{i, j_{i}}=x_{i, j_{i}}^{G}$.

Any $x_{1, j_{1}}, \ldots, x_{k, j_{k}}$ satisfy

$$
\left|C_{G}\left(x_{1, j_{1}}\right)\right| \ldots\left|C_{G}\left(x_{k, j_{k}}\right)\right| \leq\left(q^{\left(4-\frac{4}{k}-\frac{(3 k-4) \varepsilon}{k^{2}}\right) r}\right)^{k}=q^{\left(4 k-4-\left(3-\frac{4}{k}\right) \varepsilon\right) r} .
$$

Hence, according to Theorem 3.2, if $r \geq r_{1}$ then

$$
\sum_{\substack{\chi \in \operatorname{Irr} G \\ \chi \neq 1}} \frac{\left|\chi\left(x_{1, j_{1}}\right)\right| \ldots\left|\chi\left(x_{k, j_{k}}\right)\right|}{\chi(1)^{k-1}} \rightarrow 0
$$

as $|G| \rightarrow \infty$.

In particular there exists $N=N(k, \varepsilon)$ such that if $|G| \geq N$ then for any $\chi \neq 1$ we have

$$
\frac{\left|\chi\left(x_{1, j_{1}}\right)\right| \ldots\left|\chi\left(x_{k, j_{k}}\right)\right|}{\chi(1)^{k-1}} \leq 1 \text {. }
$$

Hence, if $r \geq r_{1}$ and $|G| \geq N$ then,

$$
\begin{aligned}
\sum_{\substack{\chi \in \operatorname{Irr} G \\
\chi \neq 1}} \frac{\left|\chi\left(x_{1, j_{1}}\right)\right|^{2} \cdots\left|\chi\left(x_{k, j_{k}}\right)\right|^{2}}{\chi(1)^{2 k-2}} \leq & \sum_{\substack{\chi \in \operatorname{Irr} G \\
\chi \neq 1}} \frac{\left|\chi\left(x_{1, j_{1}}\right)\right| \cdots\left|\chi\left(x_{k, j_{k}}\right)\right|}{\chi(1)^{k-1}} \\
\leq & 2 c_{1}^{1-k} q^{k \sqrt{(4 k-4) r+b}-r(k-1)+1} \\
& +c_{2}^{1-k} q^{3 k-3-\left(1.5-\frac{2}{k}\right) r \varepsilon} .
\end{aligned}
$$

The last inequality is from Theorem 3.2.

Therefore,

$$
\begin{array}{rl}
\| P_{C_{1, j}} & * \cdots * P_{C_{k, j_{k}}}-U_{G} \|_{1} \\
& \leq \sqrt{\sum_{\substack{\chi \in \operatorname{Irr} G \\
\chi \neq 1}} \frac{\left|\chi\left(x_{1, j_{1}}\right)\right|^{2} \ldots\left|\chi\left(x_{k, j_{k}}\right)\right|^{2}}{\chi(1)^{2 k-2}}} \\
& \leq \sqrt{2 c_{1}^{1-k} q^{k \sqrt{(4 k-4) r+b-r(k-1)+1}+c_{2}^{1-k} q^{3 k-3-\left(1.5-\frac{2}{k}\right) r \varepsilon}}} .
\end{array}
$$


Hence, when $r \geq r_{1}$ and $|G| \geq N$ then

$$
\begin{aligned}
& \left\|P_{W_{1,1}} * \cdots * P_{W_{k, 1}}-U_{G}\right\|_{1} \\
& \quad \leq \sum_{\substack{1 \leq j_{1} \leq m_{1} \\
1 \leq j_{k} \leq m_{k}}} \frac{\left|C_{1, j_{1}}\right| \ldots\left|C_{k, j_{k}}\right|}{\left|W_{1,1}\right| \ldots\left|W_{k, 1}\right|}\left\|P_{C_{1, j_{1}}} * \cdots * P_{C_{k, j_{k}}}-U_{G}\right\|_{1} \\
& \leq \sqrt{2 c_{1}^{1-k} q^{k \sqrt{(4 k-4) r+b}-r(k-1)+1}+c_{2}^{1-k} q^{3 k-3-\left(1.5-\frac{2}{k}\right) r \varepsilon}} .
\end{aligned}
$$

The other $2^{k}-1$ summands:

Each summand is of the form

$$
\frac{\left|W_{1, j_{1}}\right|}{\left|W_{1}\right|} \ldots \frac{\left|W_{k, j_{k}}\right|}{\left|W_{k}\right|}\left\|P_{W_{1, j_{1}}} * \cdots * P_{W_{k, j_{k}}}-U_{G}\right\|_{1},
$$

where $j_{1}, \ldots, j_{k} \in\{1,2\}$ and at least one of $j_{1}, \ldots, j_{k}$ equals 2 .

Since $\|P-Q\|_{1} \leq 2$ for distributions $P$ and $Q$, we have

$$
\frac{\left|W_{1, j_{1}}\right|}{\left|W_{1}\right|} \ldots \frac{\left|W_{k, j_{k}}\right|}{\left|W_{k}\right|}\left\|P_{W_{1, j_{1}}} * \cdots * P_{W_{k, j_{k}}}-U_{G}\right\|_{1} \leq 2 \frac{\left|W_{1, j_{1}}\right|}{\left|W_{1}\right|} \ldots \frac{\left|W_{k, j_{k}}\right|}{\left|W_{k}\right|} .
$$

Denote

$$
S=\left\{x \in G:\left|C_{G}(x)\right| \geq q^{\left(4-\frac{4}{k}-\frac{(3 k-4) \varepsilon}{k^{2}}\right) r}\right\} .
$$

Denote by $k(G)$ the number of conjugacy classes in $G$. Then $S$ is a normal subset, which splits into at most $k(G)$ conjugacy classes, and each conjugacy class is of size at most

$$
|G| q^{\left(-4+\frac{4}{k}+\frac{(3 k-4) \varepsilon}{k^{2}}\right) r} .
$$

Hence

$$
|S| \leq k(G)|G| q^{\left(-4+\frac{4}{k}+\frac{(3 k-4) \varepsilon}{k^{2}}\right) r} .
$$

According to (ii) of Lemma 2.8:

$$
k(G) \leq c_{3} q^{r}
$$

where $c_{3}$ is an absolute constant. So

$$
|S| \leq c_{3}|G| q^{\left(-3+\frac{4}{k}+\frac{(3 k-4) \varepsilon}{k^{2}}\right) r}=c_{3}|G| q^{-\left(3-\frac{4}{k}\right) r\left(1-\frac{\varepsilon}{k}\right)} .
$$

Since $W_{i, 2} \subseteq S$ we obtain

$$
\frac{\left|W_{i, 2}\right|}{|G|} \leq c_{3} q^{-\left(3-\frac{4}{k}\right) r\left(1-\frac{\varepsilon}{k}\right)}
$$

for $1 \leq i \leq k$. 
We are assuming $\frac{\left|W_{i}\right|}{|G|} \geq q^{-\left(3-\frac{4}{k}\right) r(1-\varepsilon)}$.

Hence

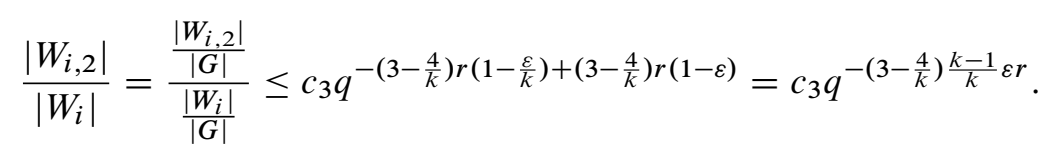

Since at least one of $j_{1}, \ldots, j_{k}$ equals 2 ,

$$
\frac{\left|W_{1, j_{1}}\right|}{\left|W_{1}\right|} \ldots \frac{\left|W_{k, j_{k}}\right|}{\left|W_{k}\right|} \leq c_{3} q^{-\left(3-\frac{4}{k}\right) \frac{k-1}{k} \varepsilon r},
$$

and so

$$
\begin{aligned}
\frac{\left|W_{1, j_{1}}\right|}{\left|W_{1}\right|} \ldots \frac{\left|W_{k, j_{k}}\right|}{\left|W_{k}\right|}\left\|P_{W_{1, j_{1}}} * \cdots * P_{W_{k, j_{k}}}-U_{G}\right\|_{1} & \leq 2 \frac{\left|W_{1, j_{1}}\right|}{\left|W_{1}\right|} \ldots \frac{\left|W_{k, j_{k}}\right|}{\left|W_{k}\right|} \\
& \leq 2 c_{3} q^{-\left(3-\frac{4}{k}\right) \frac{k-1}{k} \varepsilon r} .
\end{aligned}
$$

The sum:

When $r \geq r_{1}$ and $|G| \geq N$ we obtain

$$
\begin{aligned}
& \left\|P_{W_{1}} * \cdots * P_{W_{k}}-U_{G}\right\|_{1} \\
& \leq \sum_{\left(j_{1}, \ldots, j_{k}\right) \in\{1,2\}^{k}} \frac{\left|W_{1, j_{1}}\right|}{\left|W_{1}\right|} \cdots \frac{\left|W_{k, j_{k}}\right|}{\left|W_{k}\right|}\left\|P_{W_{1, j_{1}}} * \cdots * P_{W_{k, j_{k}}}-U_{G}\right\|_{1} \\
& \leq \sqrt{2 c_{1}^{1-k} q^{k \sqrt{(4 k-4) r+b}-r(k-1)+1}+c_{2}^{1-k} q^{3 k-3-\left(1.5-\frac{2}{k}\right) r \varepsilon}} \\
& \quad+\left(2^{k}-1\right) 2 c_{3} q^{-\left(3-\frac{4}{k}\right) \frac{k-1}{k} \varepsilon r},
\end{aligned}
$$

proving (i). Part (ii) is an immediate consequence.

We now draw conclusions for sets $w(G)$ of word values.

Theorem 3.4. Let $w \neq 1$ be a non-trivial group word. Let $G=G_{r}(q)$ be a finite simple group of Lie type of rank $r$ over the field with q elements. Then there is an integer $r_{0}(w)$, depending only on $w$, such that the following holds.

(i) If $r \geq r_{0}(w)$ and $|G| \geq N(w)$, where $N(w)$ depends only on $w$, then

$$
\left\|P_{w(G)} * P_{w(G)}-U_{G}\right\|_{1} \leq \sqrt{2 c_{1}^{-1} q^{2 \sqrt{4 r+b}-r+1}+c_{2}^{-1} q^{3-\frac{r}{3}}}+6 c_{3} q^{-\frac{r}{3}},
$$

where $c_{1}, c_{2}, c_{3}, b$ are absolute constants.

(ii) If $r \geq r_{0}(w)$ then $\left\|P_{w(G)} * P_{w(G)}-U_{G}\right\|_{1} \rightarrow 0$ as $|G| \rightarrow \infty$. 
Proof. We will deal separately with the different types of groups $G$ to show that for $r$ large enough and $|G|$ large enough we have $\frac{|w(G)|}{|G|} \geq q^{-\frac{r}{3}}$.

$G$ is not of type $A_{r}$ or ${ }^{2} A_{r}$ :

According to Theorem 2.2, there is an absolute constant $c>0$ and an integer $M(w)$, such that if $|G| \geq M(w)$ then

$$
\frac{|w(G)|}{|G|} \geq c \cdot r^{-1}
$$

There exists an absolute constant $r_{2}$ (that depends only on the absolute constant $c$ ) such that for $r \geq r_{2}$, we have $c \cdot r^{-1} \geq 2^{-\frac{r}{3}}$. So for $r \geq r_{2}$ and $|G| \geq M(w)$ we have

$$
\frac{|w(G)|}{|G|} \geq c \cdot r^{-1} \geq 2^{-\frac{r}{3}} \geq q^{-\frac{r}{3}} .
$$

$G$ is of type $A_{r}$ :

According to Theorem 2.3, if $G=\operatorname{SL}(r+1, q)$, and given $w$, there is an integer $d(w)>0$, depending only on $w$, such that

$$
\frac{|w(G)|}{|G|}>\frac{d(w)}{(r+1)^{3} q^{24+\frac{r+1}{4}}} .
$$

For $G=\operatorname{PSL}(r+1, q)$ we also have this inequality, since for a subset $S \subseteq G$ we have

$$
\frac{|\bar{S}|}{|\bar{G}|}=\frac{|\bar{S}|}{|G| /|N|} \geq \frac{|S| /|N|}{|G| /|N|}=\frac{|S|}{|G|},
$$

where $N=Z(G), \bar{G}=G / N$ and $\bar{S}=S N / N$.

So for $r$ larger than a constant $r_{3}(w)$ that depends only on $d(w)$ (and therefore only on $w$ ) we have

$$
\frac{|w(G)|}{|G|}>q^{-\frac{r}{3}} \text {. }
$$

$G$ is of type ${ }^{2} A_{r}$ :

In this case the argument is similar to the case of $A_{r}$ using Theorem 2.4 in place of Theorem 2.3. Hence there is a constant $r_{4}(w)$ such that if $r \geq r_{4}(w)$ then $\frac{|w(G)|}{|G|}>$ $q^{-\frac{r}{3}}$

\section{Conclusion:}

If $G=G_{r}(q)$ is a finite simple group of Lie type, and $w$ is a group word, there are integers $M(w)$ and $r_{2}, r_{3}(w), r_{4}(w)$ such that if $|G| \geq M(w)$ and $r \geq$ $\max \left\{r_{2}, r_{3}(w), r_{4}(w)\right\}$, then $\frac{|w(G)|}{|G|} \geq q^{-\frac{r}{3}}$.

Now we can use Theorem 3.3 with $\varepsilon=\frac{2}{3}$ and $k=2$ and obtain: 


$$
\begin{aligned}
& \text { If } r \geq \max \left\{r_{1}\left(2, \frac{2}{3}\right), r_{2}, r_{3}(w), r_{4}(w)\right\} \text { and }|G| \geq \max \left\{N\left(\frac{2}{3}\right), M(w)\right\} \text { then, } \\
& \quad\left\|P_{w(G)} * P_{w(G)}-U_{G}\right\|_{1} \leq \sqrt{2 c_{1}^{-1} q^{2 \sqrt{4 r+b}-r+1}+c_{2}^{-1} q^{3-\frac{r}{3}}}+6 c_{3} q^{-\frac{r}{3}},
\end{aligned}
$$

where $c_{1}, c_{2}, c_{3}, b$ are absolute constants.

Part (ii) is an immediate consequence.

Theorem 3.5. Let $G$ be a finite simple group of Lie type of rank $r$ over the field with $q$ elements. Let $0<\varepsilon<1$. Let $W_{1}, W_{2}$ be normal subsets such that

$$
\frac{\left|W_{i}\right|}{|G|} \geq q^{-(1-\varepsilon)} \quad \text { for } i=1,2 \text {. }
$$

Then:

(i) $\left\|P_{W_{1}} * P_{W_{2}}-U_{G}\right\|_{1} \leq \sqrt{d(r) \cdot\left(\zeta_{G}(2)-1\right)}+30 \cdot q^{-\varepsilon}$, where $d(r)$ depends only on $r$.

(ii) If $r$ is bounded, then $\left\|P_{W_{1}} * P_{W_{2}}-U_{G}\right\|_{1} \rightarrow 0$ as $|G| \rightarrow \infty$.

Proof. Partition $W_{i}$ into two subsets $W_{i, 1}$ and $W_{i, 2}: W_{i, 1}$ will be the set of all regular semisimple elements in $W_{i}$, and $W_{i, 2}$ will be the rest of the elements in $W_{i}$.

Then $W_{i, j}$ are normal subsets, and $W_{i}=W_{i, 1} \cup W_{i, 2}, W_{i, 1} \cap W_{i, 2}=\emptyset$.

Using inequality (1) in the proof of Theorem 3.3 for $k=2$ we obtain:

$$
\begin{aligned}
\left\|P_{W_{1}} * P_{W_{2}}-U_{G}\right\|_{1} \leq & \frac{\left|W_{1,1}\right|}{\left|W_{1}\right|} \frac{\left|W_{2,1}\right|}{\left|W_{2}\right|}\left\|P_{W_{1,1}} * P_{W_{2,1}}-U_{G}\right\|_{1} \\
& +\frac{\left|W_{1,1}\right|}{\left|W_{1}\right|} \frac{\left|W_{2,2}\right|}{\left|W_{2}\right|}\left\|P_{W_{1,1}} * P_{W_{2,2}}-U_{G}\right\|_{1} \\
& +\frac{\left|W_{1,2}\right|}{\left|W_{1}\right|} \frac{\left|W_{2,1}\right|}{\left|W_{2}\right|}\left\|P_{W_{1,2}} * P_{W_{2,1}}-U_{G}\right\|_{1} \\
& +\frac{\left|W_{1,2}\right|}{\left|W_{1}\right|} \frac{\left|W_{2,2}\right|}{\left|W_{2}\right|}\left\|P_{W_{1,2}} * P_{W_{2,2}}-U_{G}\right\|_{1} .
\end{aligned}
$$

We will handle the first summand differently from the other three summands.

The first summand:

$$
\frac{\left|W_{1,1}\right|}{\left|W_{1}\right|} \frac{\left|W_{2,1}\right|}{\left|W_{2}\right|}\left\|P_{W_{1,1}} * P_{W_{2,1}}-U_{G}\right\|_{1} \leq\left\|P_{W_{1,1}} * P_{W_{2,1}}-U_{G}\right\|_{1} .
$$

$W_{1,1}$ and $W_{2,1}$ are normal subsets, and hence are unions of conjugacy classes. Denote these conjugacy classes of $W_{i, 1}$ by $C_{i, 1}, \ldots, C_{i, m_{i}}$. So $W_{i, 1}=\bigcup_{j=1}^{m_{i}} C_{i, j}$.

Therefore, as in the proof of Theorem 3.3,

$$
\left\|P_{W_{1,1}} * P_{W_{2,1}}-U_{G}\right\|_{1} \leq \sum_{\substack{1 \leq j \leq m_{1} \\ 1 \leq k \leq m_{2}}} \frac{\left|C_{1, j}\right|\left|C_{2, k}\right|}{\left|W_{1,1}\right|\left|W_{2,1}\right|}\left\|P_{C_{1, j}} * P_{C_{2, k}}-U_{G}\right\|_{1} .
$$


By Lemma 2.12 there is a number $c(r)$, depending on $r$ but not on $q$, such that $|\chi(x)| \leq c(r)$ for all $\chi \in \operatorname{Irr} G$ and all regular semisimple elements $x \in G$. Using Lemma 2.6 we obtain

$$
\begin{aligned}
\left\|P_{C_{1, j}} * P_{C_{2, k}}-U_{G}\right\|_{1}^{2} & \leq \sum_{\substack{\chi \in \operatorname{Irr} G \\
\chi \neq 1}} \frac{\left|\chi\left(x_{1, j}\right)\right|^{2}\left|\chi\left(x_{2, k}\right)\right|^{2}}{\chi(1)^{2}} \\
& \leq \sum_{\substack{\chi \in \operatorname{Irr} G \\
\chi \neq 1}} \frac{c(r)^{4}}{\chi(1)^{2}}=c(r)^{4} \cdot\left(\zeta_{G}(2)-1\right),
\end{aligned}
$$

where $C_{i, j}=x_{i, j}^{G}$ in the second expression.

Thus

$$
\begin{aligned}
\left\|P_{W_{1,1}} * P_{W_{2,1}}-U_{G}\right\|_{1} & \leq \sum_{\substack{1 \leq j \leq m_{1} \\
1 \leq k \leq m_{2}}} \frac{\left|C_{1, j}\right|\left|C_{2, k}\right|}{\left|W_{1,1}\right|\left|W_{2,1}\right|}\left\|P_{C_{1, j}} * P_{C_{2, k}}-U_{G}\right\|_{1} \\
& \leq \sqrt{c(r)^{4} \cdot\left(\zeta_{G}(2)-1\right)} .
\end{aligned}
$$

The other three summands:

According to Corollary 2.11, if we denote by $r(G)$ the proportion of regular semisimple elements in $G$, then $\frac{\left|W_{i, 2}\right|}{|G|} \leq 1-r(G) \leq \frac{5}{q}$.

We assume $\frac{\left|W_{i}\right|}{|G|} \geq q^{-(1-\varepsilon)}$.

Hence

$$
\frac{\left|W_{i, 2}\right|}{\left|W_{i}\right|}=\frac{\frac{\left|W_{i, 2}\right|}{|G|}}{\frac{\left|W_{i}\right|}{|G|}} \leq 5 \cdot q^{-1+(1-\varepsilon)}=5 \cdot q^{-\varepsilon} .
$$

Since $\|P-Q\| \leq 2$ for distributions $P$ and $Q$, we have

$$
\begin{aligned}
& \frac{\left|W_{1,1}\right|}{\left|W_{1}\right|} \frac{\left|W_{2,2}\right|}{\left|W_{2}\right|}\left\|P_{W_{1,1}} * P_{W_{2,2}}-U_{G}\right\|_{1}+\frac{\left|W_{1,2}\right|}{\left|W_{1}\right|} \frac{\left|W_{2,1}\right|}{\left|W_{2}\right|}\left\|P_{W_{1,2}} * P_{W_{2,1}}-U_{G}\right\|_{1} \\
&+\frac{\left|W_{1,2}\right|}{\left|W_{1}\right|} \frac{\left|W_{2,2}\right|}{\left|W_{2}\right|}\left\|P_{W_{1,2}} * P_{W_{2,2}}-U_{G}\right\|_{1} \leq 2 \frac{\left|W_{2,2}\right|}{\left|W_{2}\right|}+2 \frac{\left|W_{1,2}\right|}{\left|W_{1}\right|}+2 \frac{\left|W_{1,2}\right|}{\left|W_{1}\right|} \\
& \leq 6 \cdot 5 \cdot q^{-\varepsilon} .
\end{aligned}
$$

The sum:

$$
\left\|P_{W_{1}} * P_{W_{2}}-U_{G}\right\|_{1} \leq \sqrt{d(r) \cdot\left(\zeta_{G}(2)-1\right)}+30 \cdot q^{-\varepsilon},
$$

proving (i).

In (ii) we assume that $r$ is bounded, and so $d(r)$ is bounded. We also know from Theorem 2.9 that $\zeta_{G}(2) \rightarrow 1$ as $|G| \rightarrow \infty$. If $r$ is bounded and $|G| \rightarrow \infty$ then $q \rightarrow \infty$, proving (ii). 
Notice that with these tools we cannot prove a better result for $k$ normal subsets instead of 2.

For sets of the form $W=w(G)$ we now obtain:

Theorem 3.6. Let $w \neq 1$ be a non-trivial group word. Let $G=G_{r}(q)$ be a finite simple group of Lie type of rank $r$ over the field with q elements. Assume there exists $r_{0}(w)$, that may depend on $w$, such that $r \leq r_{0}(w)$. Then:

(i) There exist constants $N(w), d(w)$, depending only on $w$, such that if $|G| \geq$ $N(w)$, then

$$
\left\|P_{w(G)} * P_{w(G)}-U_{G}\right\|_{1} \leq \sqrt{d(w) \cdot\left(\zeta_{G}(2)-1\right)}+30 \cdot q^{-\frac{1}{2}} .
$$

(ii) $\left\|P_{w(G)} * P_{w(G)}-U_{G}\right\|_{1} \rightarrow 0$ as $|G| \rightarrow \infty$.

Proof. We are assuming that $r$ is bounded by $r_{0}(w)$, so according to Theorem 2.1 there exists a constant $c(w)>0$ that depends only on $w$ such that $\frac{|w(G)|}{|G|} \geq c(w)$. Since we are assuming $r$ is bounded, we have $q \rightarrow \infty$ as $|G| \rightarrow \infty$. So there exists $N(w)$ such that if $|G| \geq N(w)$ then $q^{-\frac{1}{2}} \leq c(w)$. So if $|G| \geq N(w)$ then $\frac{|w(G)|}{|G|} \geq c(w) \geq q^{-\frac{1}{2}}$.

We now apply Theorem 3.5 with $\varepsilon=1 / 2$. Since $r \leq r_{0}(w)$ and $|G| \geq N(w)$, we have

$$
\left\|P_{w(G)} * P_{w(G)}-U_{G}\right\|_{1} \leq \sqrt{d(w) \cdot\left(\zeta_{G}(2)-1\right)}+30 \cdot q^{-\frac{1}{2}},
$$

where $d(w)$ depends only on $w$, proving (i).

Part (ii) also follows since $\zeta_{G}(2) \rightarrow 1$ as $|G| \rightarrow \infty$, and since $q \rightarrow \infty$ as $|G| \rightarrow \infty$.

Theorem 3.7. Let $w \neq 1$ be a non-trivial group word and let $G$ be a finite simple group. Then $\left\|P_{w(G)} * P_{w(G)}-U_{G}\right\|_{1} \rightarrow 0$ as $|G| \rightarrow \infty$.

Proof. According to 1.17 of [LaSh2], $\left\|P_{w(G)} * P_{w(G)}-U_{G}\right\|_{1} \rightarrow 0$ as $|G| \rightarrow \infty$ if $G$ is an alternating group.

For groups of Lie type use Theorems 3.4 and 3.6 to obtain the result.

Since $|G| \rightarrow \infty$ we can omit the sporadic groups.

According to the classification of finite simple groups this covers all of the finite simple group.

Corollary 3.8. Let $k$ be a positive integer and let $w=x^{k}$. Let $G$ be a finite simple group. Then $\left\|P_{w(G)} * P_{w(G)}-U_{G}\right\|_{1} \rightarrow 0$ as $|G| \rightarrow \infty$. 


\section{References}

[Di1] P. Diaconis, Group representations in probability and statistics. IMS Lecture NotesMonograph Ser. 11, Institute of Mathematical Statistics, Hayward, CA, 1988. Zbl 0695.60012 MR 0964069

[Di2] P. Diaconis, Random walks on groups: characters and geometry. In Groups St Andrews 2001 in Oxford, Vol. I, London Math. Soc. Lecture Note Ser. 304, Cambridge University Press, Cambridge 2003, 120-142. Zbl 1064.20071 MR 2051523

[DiS] P. Diaconis and M. Shahshahani, Generating a random permutation with random transpositions. Z. Wahrsch. verw. Gebiete 57 (1981), 159-179. Zbl 0485.60006 MR 626813

[FuGu] J. Fulman and R. Guralnick, Bounds on the number and sizes of conjugacy classes in finite Chevalley groups with applications to derangements. Preprint 2009. arXiv:0902.22381

[GaSh] S. Garion and A. Shalev, Commutator maps, measure preservation, and $T$-systems. Trans. Amer. Math. Soc. 361 (2009), 4631-4651. Zbl 1182.20015 MR 2506422

[Go] W. T. Gowers, Quasirandom groups. Combin. Probab. Comput. 17 (2008), 363-387. Zbl 1191.20016 MR 2410393

[GuLu] R. M. Guralnick and F. Lübeck, On $p$-singular elements in Chevalley groups in characteristic $p$. In Groups and computation III, Ohio State Univ. Math. Res. Inst. Publ. 8, de Gruyter, Berlin 2001, 169-182. Zbl 1001.20045 MR 1829478

[JaLi] G. James and M. Liebeck, Representations and characters of groups. Cambridge Math. Textbooks, Cambridge University Press, Cambridge 1993. Zbl 0792.20006 MR 1237401

[LanSe] V. Landazuri and G. M. Seitz, On the minimal degrees of projective representations of the finite Chevalley groups. J. Algebra 32 (1974), 418-443. Zbl 0325.20008 MR 0360852

[La] M. Larsen, Word maps have large image. Israel J. Math. 139 (2004), 149-156. Zbl 1130.20310 MR 2041227

[LaSh1] M. Larsen and A. Shalev, Word maps and Waring type problems. J. Amer. Math. Soc. 22 (2009), 437-466. MR 2476780

[LaSh2] M. Larsen and A. Shalev, Characters of symmetric groups: sharp bounds and applications. Invent. Math. 174 (2008), 645-687. Zbl 1166.20009 MR 2453603

[LiSh1] M. W. Liebeck and A. Shalev, Diameters of finite simple groups: sharp bounds and applications. Ann. of Math. (2) 154 (2001), 383-406. Zbl 1003.20014 MR 1865975

[LiSh2] M. W. Liebeck and A. Shalev, Fuchsian groups, finite simple groups and representation varieties. Invent. Math. 159 (2005), 317-367. Zbl 1134.20059 MR 2116277

[LiSh3] M. W. Liebeck and A. Shalev, Character degrees and random walks in finite groups of Lie type. Proc. London Math. Soc. (3) 90 (2005), 61-86. Zbl 1077.20020 MR 2107038

[Lul] N. Lulov, Random walks on symmetric groups generated by conjugacy classes. Ph.D. Thesis, Harvard University, Cambridge, MA, 1996. 
[Lus] G. Lusztig, Characters of reductive groups over a finite field. Ann. of Math. Studies, Princeton University Press, Princeton 1984. Zbl 0556.20033 MR 0742472

[NiPy] N. Nikolov and L. Pyber, Product decompositions of quasirandom groups and a Jordan type theorem. Europ. J. Math., to appear.

[Ser] J.-P. Serre, Linear representations of finite groups. Graduate Texts in Math. 42, Springer-Verlag, New York 1977. Zbl 0355.20006 MR 0450380

[Sh1] A. Shalev, Mixing and generation in simple groups. J. Algebra 319 (2008), 30753086. Zbl 1146.20057 MR 2397424

[Sh] A. Shalev, Word maps, conjugacy classes, and a noncommutative Waring-type theorem. Ann. of Math. (2) 170 (2009), 1383-1416. Zbl 05710189 MR 2600876

Received April 30, 2009; revised August 25, 2009

G. Schul, A. Shalev, Institute of Mathematics, The Hebrew University, Jerusalem 91904, Israel

E-mail: gili.schul@mail.huji.ac.il; shalev@math.huji.ac.il 